\title{
ACESSIBILIDADE DO HOMEM AOS SERVIÇOS DA ATENÇÃO BÁSICA: UMA APROXIMAÇÃO COM A BIOÉTICA DA PROTEÇÃO*
}

Doane Martins da Silva ${ }^{1}$, Tatiane Oliveira de Souza ${ }^{1}$, Maristela Viana Lima², Sérgio Donha Yarid ${ }^{3}$, Edite Lago da Silva Sena ${ }^{4}$

RESUMO: Estudo bibliográfico objetivou analisar estudos sobre a temática da acessibilidade do homem aos serviços da Atenção Básica após a implementação da Política Nacional de Atenção Integral à Saúde do Homem, dialogando essa temática com a bioética da proteção. Foram analisados 10 estudos do período de 2009 a 2012 publicados nas bases de dados indexadas na Biblioteca Virtual em Saúde. A discussão das informações estruturou-se em duas temáticas: Dificuldades de acessibilidade aos serviços da Atenção Básica reveladas pelos homens e Acessibilidade do homem aos serviços da Atenção Básica: um problema ético. $\mathrm{O}$ material revisado evidencia a existência de fatores relacionados ao funcionamento dos serviços e ao estereótipo de masculinidade construído culturalmente os quais dificultam a acessibilidade do homem aos serviços. Considera-se salutar a abordagem da bioética no contexto desta acessibilidade, pois a temática representa, efetivamente, um problema ético no campo da saúde pública.

DESCRITORES: Saúde do homem; Acesso aos serviços de saúde; Atenção primária à saúde; Bioética.

\section{MEN'S ACCESSIBILITY TO THE PRIMARY CARE SERVICES: AN APROXIMATION WITH THE BIOETHICS OF PROTECTION}

ABSTRACT: This bibliographic study aimed to analyze studies on the issue of men's accessibility to the Primary Care Services following the implementation of the National Comprehensive Healthcare Policy for Men, engaging this topic with the bioethics of protection. 10 studies were analyzed, from the period 2009-2012, published in the databases indexed in the Virtual Health Library. The discussion of the information was structured in two themes: Difficulties in accessing the Primary Care Services revealed by the men and The man's accessibility to the Primary Care Services: an ethical problem. The material reviewed evidences the existence of factors related to the functioning of the services and to the stereotype of masculinity constructed culturally, both of which hinder men's access to the services. It is considered beneficial to address bio-ethics in the context of this accessibility, as the issue represents, effectively, an ethical problem in the field of public health.

DESCRIPTORS: Men's health; Access to the health services; Primary healthcare; Bio-ethics.

\section{ACCESIBILIDAD DEL HOMBRE A LOS SERVICIOS DE LA ATENCIÓN BÁSICA: UNA APROXIMACIÓN CON LA BIOÉTICA DE LA PROTECCIÓN}

RESUMEN: Artículo bibliográfico cuya finalidad fue analizar estudios sobre la temática de la accesibilidad del hombre a los servicios de la Atención Básica después de la implementación de la Política Nacional de Atención Integral a la Salud del Hombre, estableciendo un diálogo entre esa temática y la bioética de la protección. Fueron analizados 10 estudios del periodo de 2009 a 2012 publicados en las bases de datos indexadas en la Biblioteca Virtual en Salud. La discusión de las informaciones se estructuró en dos temáticas: Dificultades de accesibilidad a los servicios de la Atención Básica reveladas por los hombres y Accesibilidad del hombre a los servicios de la Atención Básica: un problema ético. El material revisado evidencia la existencia de factores asociados al funcionamiento de los servicios y al estereotipo de masculinidad construido culturalmente, los cuales dificultan la accesibilidad del hombre a los servicios. Se considera salutar el abordaje de la bioética en el contexto de esta accesibilidad, pues la temática representa, efectivamente, un problema ético en el ámbito de la salud pública.

DESCRIPTORES: Salud del hombre; Acceso a los servicios de salud; Atención primaria a la salud; Bioética.

\footnotetext{
*Trabalho elaborado na disciplina Bioética em Ciência da Saúde do Programa de Pós-Graduação em Enfermagem e Saúde - Mestrado da Universidade Estadual do Sudoeste da Bahia - UESB, Campus de Jequié - Bahia.

${ }^{1}$ Enfermeira. Mestranda pelo Programa de Pós-Graduação em Enfermagem e Saúde -PPGES UESB.

${ }^{2}$ Psicóloga. Especialista em Saúde Mental.

${ }^{3}$ Odontólogo. Doutor em Odontologia Preventiva e Social. Professor do Curso de Graduação em Odontologia e do PPGES UESB.

${ }^{4}$ Enfermeira. Doutora em Enfermagem. Professora do Curso de Graduação em Enfermagem e do PPGES UESB.
} 


\section{INTRODUÇÃO}

A Constituição Federal Brasileira, promulgada em 1988, considera em seu artigo 196 ser a a saúde um direito de todos e um dever do Estado ${ }^{(1)}$. Essa mesma Constituição colocou as bases do Sistema Único de Saúde (SUS), que foi posteriormente regulamentado pelas Leis n. 8.080/90 e n. 8.142/90 ${ }^{(2-3)}$. A primeira lei dispõe acerca das condições para a promoção, proteção, recuperação da saúde, bem como a organização e funcionamento dos serviços e estabelece, entre os princípios do SUS, a universalidade de acesso $^{(2)}$. Já a Lei n. 8.142 trata da participação da comunidade e sobre as transferências dos recursos financeiros entre as esferas de governo na área de saúde ${ }^{(3)}$.

Desde que a saúde no país foi instituída como um direito de todos e dever do Estado, operada por meio do SUS, vem-se atribuindo ênfase às modificações nesse modelo de atenção à saúde, com o intuito de priorizar o nível da Atenção Básica ${ }^{(4)}$. Destaca-se que, embora os termos 'Atenção Primária à Saúde' (APS) e 'Atenção Básica' (AB) sejam utilizados indistintamente, há o reconhecimento e a utilização internacional da terminologia $\mathrm{APS}^{(5)}$. No Brasil, o Conselho Nacional de Secretários de Saúde adotou a nomenclatura Atenção Básica para enfatizar a reorientação do modelo assistencial ${ }^{(6)}$. Desta forma, no presente estudo optou-se por utilizar ambas as terminologias tal como são apresentadas nas produções científicas analisadas.

A APS é a porta preferencial para o sistema de saúde no país e representa um esforço para que o SUS se consolide, tornando-se mais eficiente, fortalecendo os vínculos entre serviço e população, contribuindo para a universalização do acesso e para a garantia da integralidade e equidade da assistência ${ }^{(7)}$. No entanto, quando se reporta à acessibilidade do homem aos serviços da $A B$ verifica-se que esta permanece como um desafio para a efetivação do direito universal à saúde. Para fazer frente a este desafio, é necessário que esta problemática faça parte de discussões no campo da bioética, já que representa um problema ético no campo da saúde pública.

A acessibilidade consiste na capacidade do sistema de saúde quanto à produção e oferta de serviços aptos à atrair, e ao mesmo tempo responder as necessidades de saúde de determinada população ${ }^{(8)}$. Nesse contexto, destaca-se a ocorrência de baixa acessibilidade do homem aos serviços da $\mathrm{AB}$, o que sugere sua vulnerabilidade. Desta forma, torna-se relevante problema de saúde pública, já que a busca pelos ser- viços de saúde pela população masculina, quando existe, está atrelada a um quadro clínico de morbidade cronificado, onerando significativamente o SUS.

Assim, com o propósito de promover a melhoria das condições de saúde da população masculina, por meio do enfrentamento racional dos fatores de risco e mediante a facilitação ao acesso às ações e aos serviços de assistência integral à saúde, foi instituída, no âmbito do SUS, por meio da Portaria n. 1.944/09(9), a Política Nacional de Atenção Integral à Saúde do Homem (PNAISH).

A partir destas considerações, definiu-se como objetivo deste estudo analisar pesquisas que abordem a temática da acessibilidade do homem aos serviços da $\mathrm{AB}$ após a implementação da PNAISH, dialogando essa temática com a bioética da proteção.

\section{MÉTODO}

Trata-se de um estudo bibliográfico. O levantamento das pesquisas foi realizado durante os meses de maio a junho de 2012, nas bases de dados indexadas na Biblioteca Virtual em Saúde (BVS). Utilizaram-se os seguintes descritores agrupados: saúde do homem, atenção primária à saúde, acesso aos serviços de saúde e bioética da proteção. Localizou-se o quantitativo de 396 artigos.

Os critérios de inclusão foram: artigos de pesquisa; disponíveis na íntegra online; no idioma português, já que a PNAISH é uma política brasileira. E como critérios de exclusão: artigos sem resumo na base de dados ou incompletos. O recorte temporal utilizado foi de 2009, em virtude do marco que instituiu a PNAISH, até junho 2012. Foi realizada, numa primeira etapa, a leitura atenta dos títulos, resumos e palavras-chave/ descritores, para a identificação e adesão à proposta do estudo. Na segunda etapa, foi realizada leitura atenta dos conteúdos da publicação, identificando e transcrevendo as informações de interesse em formulário próprio.

Foram delimitados os artigos a serem interpretados em um total de 10 artigos que abordam a acessibilidade do homem aos serviços da $\mathrm{AB}$ após a implementação da PNAISH, dialogando essa temática com a bioética da proteção.

\section{RESULTADOS}

Dos 10 artigos selecionados, verificou-se que em 2009, ano de instituição da PNAISH, não foi encontrado estudo publicado nas bases de dados indexadas na BVS. No entanto, não pode ser descartada a existência 
de artigos não atingidos pelos critérios de busca, uma vez que autores podem não ter optado por esses descritores em suas pesquisas; porém, acredita-se que o agrupamento de descritores ajudou no direcionamento desta pesquisa e abarcou uma vasta produção com possível relação com o foco eleito.

De modo geral, a produção demonstra tendência de crescimento, pois observou-se que todos os artigos selecionados foram publicados entre os anos de 2010 e 2011, inferindo-se uma preocupação por parte dos pesquisadores em realizarem estudos que tratem da acessibilidade do homem aos serviços de AB após a implementação da PNAISH. Entretanto, é valido ressaltar que no ano de 2012, até o mês de junho, período final do levantamento de artigos, não se teve produção científica acerca da temática em questão.

Com suporte nos conteúdos dos dez artigos, foram categorizadas duas temáticas: Dificuldades de acessibilidade aos serviços da Atenção Básica reveladas pelos homens e Acessibilidade do homem aos serviços da Atenção Básica: um problema ético.

\section{DISCUSSÃO}

\section{Dificuldades de acessibilidade aos serviços da Aten- ção Básica reveladas pelos homens}

Dentre os estudos analisados, um deles ${ }^{(10)}$ aponta que a adequação dos serviços da $\mathrm{AB}$ às demandas do homem constitui-se num desafio para o sistema público de saúde e, um dos caminhos para se atingir a melhoria dos serviços, pode ser a escuta aos usuários possibilitando conhecer a satisfação ou insatisfação destes frente ao atendimento que lhes é prestado.

Nesse contexto, o acolhimento, a humanização, ancorada na comunicação e na resolutividade foram sentidos atribuídos pelos usuários a uma assistência de qualidade em dois estudos analisados ${ }^{(10-11)}$.

Em geral, esses sentidos se articulam com a Política Nacional de Humanização (PNH), que concebe atendimento humanizado como aquele que, entre outros aspectos, se baseia na escuta qualificada, ampliação dos processos comunicacionais e resolutividade ${ }^{(12)}$.

Estudos se reportam aos diversos fatores que dificultam a acessibilidade do homem aos serviços da $\mathrm{AB}$, envolvendo dificuldades de se equacionar a oferta dos serviços com a demanda de atendimento; a ideia de que o atendimento particular é melhor; a existência da prática de um atendimento facilitado para aqueles que são conhecidos dos profissionais e o acolhimento deficiente nos serviços ${ }^{(10-11)}$.

A este respeito, outro estudo elegido acrescenta outros fatores, a saber: demora para marcação de exames especializados; insatisfação quanto à carência de profissionais e de especialidades médicas; longo intervalo entre marcação de atendimento e a resolutividade da necessidade de saúde apresentada pelo usuário $^{(13)}$. Nesse sentido, cabe ressaltar que a $\mathrm{AB}$ por ser a principal porta de entrada para a assistência à saúde, tem um papel fundamental na resolutividade das demandas dos usuários, bem como na realização de procedimentos necessários, a fim de promover a reabilitação da saúde ${ }^{(14)}$.

Para minimizar esses problemas, a PNH traz como uma de suas diretrizes a otimização dos serviços, com o intuito de reduzir as filas e, consequentemente, o tempo de espera para obter assistência médica ${ }^{(12)}$. No entanto, apesar de instituída a PNH, a espera em filas por atendimento nas unidades básicas de saúde é apontada como fator contribuinte para a pouca frequência com que o público masculino usufrui de tais serviços. Além disso, os horários de funcionamento destes serviços são incompatíveis com a jornada de trabalho dos indivíduos inseridos no mercado formal, o que dificulta a acessibilidade do homem, pois existe o receio em serem prejudicados por se ausentarem do trabalho para buscar assistência à saúde ${ }^{(15)}$.

Neste sentido, acredita-se ser possível aumentar a demanda do homem aos serviços da AB se houvesse horário de extensão noturna, por exemplo, das 17 às 22 horas. Faz-se necessário ressaltar que os programas de saúde disponibilizados na APS no Brasil são historicamente voltados para o grupo materno-infantil e para os idosos, como aponta outro estudo selecionado ${ }^{(7)}$. Portanto, a ausência de serviços específicos de cuidados para homens alimenta a ideia de um espaço ocupado e reservado às mulheres. Isso acaba por reforçar a relação historicamente mais significativa das mulheres com os serviços de assistência à saúde e não identificação do homem com estes espaços, como apontado por estudo ${ }^{(16)}$.

Junto a isso, problematiza-se o fato de que o não reconhecimento da singularidade do homem por parte dos próprios serviços de saúde pode trazer dificuldades em incorporar esses como protagonistas de seus cuidados $^{(17)}$. Nesse contexto, estudo corrobora com essa ideia e aponta que ocorre nos serviços da $\mathrm{AB}$ uma invisibilidade do homem como alvo de intervenção em saúde, expressa sobretudo na falta de programas e atendimentos direcionados aos homens; dificuldades para acessar os serviços ou de acolhimento às deman- 
das; e como sujeitos de cuidado, que, pela expectativa dos profissionais de que o homem não cuida de si nem de outras pessoas, não são estimulados às práticas de prevenção e promoção de saúde ${ }^{(7)}$.

Além disso, outro fator que merece destaque é a forma decorativa como os funcionários organizam as unidades, tornando-as cada vez mais um espaço aparentemente feminino $^{(7)}$. De fato, os homens consideram o ambiente das unidades básicas de saúde feminilizado, o que provoca neles a sensação de não pertencimento àquele espaço ${ }^{(18)}$.

Nas avaliações negativas acerca do atendimento recebido pelos homens nos serviços da $\mathrm{AB}$, a diversidade de fatores colocados perpassa pelas duas dimensões da acessibilidade definidas por Donabedian ${ }^{(8)}$ as quais estão em contínua interação sócio-organizacional e a geográfica. A primeira engloba todos os fatores que facilitam ou dificultam o uso dos serviços de saúde pelos usuários para chegarem o atendimento, exceto os de ordem geográfica, os quais são identificados pela distância e o tempo necessário de locomoção para a utilização destes.

Nessa perspectiva, cabe salientar que a PNAISH ${ }^{(9)}$ trata das temáticas que envolvem as barreiras sócioculturais e institucionais que interferem na acessibilidade da população masculina aos serviços da APS e busca garantir que o primeiro acesso dessa população ao sistema de saúde aconteça através da $A B$, a fim de resguardar a prevenção e a promoção como eixos necessários e fundamentais de intervenção.

A partir desta vertente, acredita-se numa (re) construção das relações entre homens e os serviços da AB. E, ainda, para fazer frente ao desafio da concretização da acessibilidade do homem aos referidos serviços, apresenta-se a necessidade de que esta temática faça parte de discussões no campo da bioética.

\section{Acessibilidade do homem aos serviços da Atenção Básica: um problema ético}

A universalidade de acesso aos serviços de saúde, em todos os níveis de assistência, é incorporada como um dos princípios fundamentais do SUS, e constitui-se de fato, em princípio de alto teor bioético. Embora se reconheça os inúmeros esforços para assegurar as prerrogativas do SUS e garantir seu funcionamento, conforme sua base teórica e conquista legal, a universalidade proposta, para ser real, deve contemplar não apenas a intenção, mas principalmente a efetividade, como aponta um estudo selecionado ${ }^{(19)}$.

Nesse contexto, identificamos como pertinente que a problemática da acessibilidade do homem aos serviços da AB faça parte de discussões no campo da bioética, pois representa, efetivamente, um problema ético no campo da saúde pública.

Entende-se por problema ético as questões ou as implicações éticas de ocorrências comuns na prática da atenção à saúde nas unidades básicas, não configurando, necessariamente, um dilema, mas sim aspectos éticos que permeiam circunstâncias comuns da prática diária da atenção à saúde ${ }^{(20)}$. Dessa forma, percebemos a importância de que a gama de fatores que dificultam a acessibilidade da população masculina aos serviços da $\mathrm{AB}$ apontados nos estudos analisados ${ }^{(7,10-11,13,16-17)}$, concentram-se em torno da demanda e dos processos de trabalho nos serviços, podendo ser pensados enquanto problemas éticos.

Nesse sentido, estudo revela que estes problemas exigem um equacionamento ético que deve ser refletido a partir da humanização da assistência, pois se reconhece que a acessibilidade faz parte desta humanização, sendo necessário que ocorra a criação de vínculo e acolhimento para integrar o usuário ao serviço ${ }^{(21)}$. Nesse contexto, outros estudos revelam que como dispositivo de uma política de humanização dos serviços de saúde, o acolhimento traduz a ação humana de reconhecer a dimensão subjetiva do ser humano, considerando-o como sujeito histórico, social e cultural. Portanto, essa estratégia pretende ultrapassar os limites técnico-operacionais de uma atividade programada e tanger as esferas dos valores éticos ${ }^{(22)}$.

A este respeito, autoresapontam que os problemas éticos da saúde pública, que neste estudo diz respeito à acessibilidade do homem aos serviços da $\mathrm{AB}$, podem ser enfrentados por meio de uma nova concepção da bioética, a bioética da proteção. A ação protetora do Estado deve garantir o direito social do acesso aos serviços públicos de saúde, com qualidade e respeito aos direitos humanos fundamentais ${ }^{(23)}$.

A bioética da proteção trata de uma proposta recente, emergindo a partir da aplicação do corpo teórico-prático da bioética tradicional, tentando transformá-lo e adaptá-lo à situações-problemas provenientes dos serviços de saúde, sobretudo no âmbito da saúde pública $^{(24)}$.

Nessa perspectiva, há interface da bioética com a saúde pública, capaz de contribuir de forma expressiva com o fornecimento de critérios éticos que permitam saber a destinação e aplicação de recursos, além de buscar garantir a proteção das populações vulneráveis, conforme evidencia estudo selecionado $^{(25)}$. E, destaca-se que a ocorrência de 
uma baixa acessibilidade do homem aos serviços da AB sugere uma vulnerabilidade desses indivíduos.

A Bioética oferece subsídios teóricos e práticos para dirimir conflitos de interesses e valores que se apresentam na saúde pública. E ainda, ressalta-se quão importantes são as contribuições bioéticas para subsidiar análises e intervenções relacionadas à gestão, à estrutura, à alocação de recursos destinados à área da saúde e demais demandas presentes no cotidiano da saúde pública ${ }^{(26)}$.

Diante destas considerações, ressalta-se que a reflexão ética acerca da acessibilidade aos serviços de saúde torna-se essencial para superar os limites, reavaliar as atitudes e efetivamente assegurar a acessibilidade do homem a estes serviços.

\section{CONSIDERAÇÕES FINAIS}

O material revisado evidencia que a produção sobre a temática da acessibilidade da população masculina aos serviços da $\mathrm{AB}$ transversalizando com a bioética da proteção cadastrada nas bases de dados ainda é pequena.

A revisão bibliográfica publicada nos últimos quatro anos permitiu evidenciar que existe uma gama de fatores relacionados ao funcionamento dos serviços e ao estereótipo de masculinidade construído culturalmente que dificultam a acessibilidade dos homens aos serviços.

Neste contexto, considera-se salutar a abordagem da bioética da proteção no contexto da acessibilidade do homem aos serviços da $A B$, visto que permite contribuir para a reflexão acerca de problemas éticos que permeiam circunstâncias comuns da prática diária da atenção à saúde.

Por fim, os resultados deste estudo podem auxiliar na discussão a respeito dos entraves que dificultam a acessibilidade do homem aos serviços da $\mathrm{AB}$, oferecendo a possibilidade de subsidiar novas discussões referentes a esta temática, além de permitir o repensar crítico acerca da pertinência à pauta da discussão bioética do problema da acessibilidade à atenção em saúde.

\section{REFERÊNCIAS}

1. Brasil. Constituição da República Federativa do Brasil. Brasília-DF: Senado Federal. Centro Gráfico; 1988.

2. Brasil. Lei n. 8.080, de 19 de setembro de 1990. Dispõe sobre as condições para a promoção e recuperação da saúde, a organização e o funcionamento dos serviços correspondentes e dá outras providências. Diário oficial da República Federativa do Brasil, Brasília, 19 set. 1990a.
3. Brasil. Lei n. 8.142, de 28 de dezembro de 1990. Dispõe sobre a participação da comunidade na gestão do Sistema Único de Saúde (SUS) e sobre as transferências intergovernamentais de recursos financeiros na área da saúde e dá outras providências. Diário oficial da República Federativa do Brasil, Brasília, 28 dez. 1990b.

4. Mendes AN, Marques RM. O papel e as consequências dos incentivos como estratégia de financiamento das ações de saúde. In: Organização Pan-Americana da Saúde; Organização Mundial da Saúde; Brasil. Ministério da Saúde. Projeto de Desenvolvimento de Sistemas e Serviços de Saúde. (Série técnica Projeto de Desenvolvimento de Sistemas e Serviços de Saúde, 4). Brasília: Organização Pan-Americana da Saúde; 2003.

5. Starfield B. Atenção primária: equilíbrio entre necessidades de saúde, serviços e tecnologia. Brasília: Ministério da Saúde/UNESCO; 2004. p. 71-102.

6. Fausto MCR, Matta GC. Atenção Primária à Saúde: histórico e perspectivas. In: Morosini MVGC, Corbo AD, organizadoras. Modelos de atenção e a saúde da família. Rio de Janeiro: Ministério da Saúde, Fundação Oswaldo Cruz, EscolaPolitécnicadeSaúde Joaquim Venâncio;2007.p.43-67.

7. Couto MT, Pinheiro TF, Valença O, Machin R, Silva GSN, Gomes R, et al. O homem na atenção primária à saúde: discutindo (in)visibilidade a partir da perspectiva de gênero. Interface-Comunic., Saude, Educ. 2010;14(33):255-70.

8. Donabedian A. Los espacios de la salud: aspectos fundamentales de la organización de la atención médica. México: Editora Biblioteca de la Salud; 1988.

9. Ministério da Saúde (BR). Política Nacional de Atenção Integral à Saúde do Homem: princípios e diretrizes. Brasília: Ministério da Saúde; 2008.

10. Gomes R, Rebello LEFS, Nascimento EF, Deslandes $\mathrm{SF}$, Moreira MCN. A atenção básica à saúde do homem sob a ótica do usuário: um estudo qualitativo em três serviços do Rio de Janeiro. Ciênc. saúde colet. 2011;16(11):4513-21.

11. Gomes R, Schraiber LB, Couto MT, Valença OAA, Silva GSN, Figueiredo WS, et al. $\mathrm{O}$ atendimento à saúde de homens: estudo qualitativo em quatro estados brasileiros. Physis. 2011;21(1):113-28.

12. Ministério da Saúde (BR). Humaniza-SUS: Política Nacional de Humanização. A humanização como eixo norteador das práticas de atenção e gestão em todas as instâncias do SUS. Brasília: Ministério da Saúde; 2004. 
13. Brito RS, Santos DLA, Maciel PSO. Olhar masculino acerca do atendimento na estratégia saúde da família. Rev. Rene. 2010;11(4):135-42.

14. Oliveira MM, Coimbra VCC, Kantorski LP, Jardim VMR, Heck RM, Ceolin T. Acesso dos usuários de uma unidade de Saúde da Família a serviços complementares. Cogitare enferm. 2009;14(1):30-6.

15. Gomes R, Nascimento EF, Araújo FC. Porque os homens buscam menos os serviços de saúde do que as mulheres? As explicações de homens com baixa escolaridade e homens com ensino superior. Cad. Saúde Pública. 2007;23(3):565-74.

16. Toneli MJF, Souza MGC, Muller RCF. Masculinidades e práticas de saúde: retratos da experiência de pesquisa em Florianópolis/SC. Physis. 2010;20(3):973-94.

17. Gomes R, Moreira MCN, Nascimento EF, Rebello LEFS, Couto MT, Schraiber LB. Os homens não vêm! Ausência e/ou invisibilidade masculina na atenção primária. Ciênc. saúde colet. 2011;16(Suppl.1):983-92.

18. Figueiredo W. Assistência à Saúde do homem: um desafio para os serviços de atenção primária. Ciênc saúde colet. 2005;10(1):105-9.

19. Andrade EM, Andrade EO. O SUS e o direito à saúde do brasileiro: leitura de seus princípios, com ênfase na universalidade da cobertura. Revista Bioética. 2010;18(1):61-74.

20. Sugarman J. Ethics in primary care. New York: McGraaw-Hill; 2000. p. xiii-xvi.

21. Junges JR. Bioética da atenção primária à saúde. Revista da AMRIGS. 2011;55(1):88-90.

22. Brehmer LCF, Verdi M. Acolhimento na Atenção Básica: reflexões éticas sobre a Atenção à Saúde dos usuários. Ciênc. saúde colet. 2010;15(suppl.3):3569-78 .

23. Schramm FR, Kottow M. Principios bioéticos em salud pública: limitaciones e propuestas. Cad. Saúde Pública. 2001;17(4):949-56

24. Schramm FR. Bioética da Proteção: ferramenta válida para enfrentar problemas morais na era da globalização. Revista Bioética. 2008;16(1):11-23.

25. Petry P, Bonamigo EL, Conte K, Schlemper Junior BR. Comitê de Bioética: uma proposta para a atenção básica à saúde. Revista - Centro Universitário São Camilo. 2010;4(3):258-68.
26. Fortes PAC, Zoboli ELCP. Bioética e Saúde Pública. São Paulo: Loyola; 2003.

Cogitare Enferm. 2013 Jul/Set; 18(3):573-8 